\title{
A pipeline for differential proteomics in unsequenced species
}

Şule Yılmaz ${ }^{1,2,3}$, Bjorn Victor ${ }^{4}$, Niels Hulstaert ${ }^{1,2,3}$, Elien Vandermarliere ${ }^{1,2,3}$, Harald Barsnes $^{5}$, Sven Degroeve ${ }^{1,2,3}$, Surya Gupta ${ }^{1,2,3}$, Adriaan Sticker ${ }^{1,2,3,6}$, Sarah Gabriël ${ }^{4}$, Pierre Dorny $^{4}$, Magnus Palmblad ${ }^{7}$, Lennart Martens ${ }^{1,2,3^{*}}$

${ }^{1}$ Medical Biotechnology Center, VIB, 9000 Ghent, Belgium

${ }^{2}$ Department of Biochemistry, Ghent University, 9000 Ghent, Belgium

${ }^{3}$ Bioinformatics Institute Ghent, Ghent University, 9000 Ghent, Belgium

${ }^{4}$ Veterinary Helminthology Unit, Department of Biomedical Sciences, Institute of Tropical Medicine, Antwerp, Belgium

${ }^{5}$ Proteomics Unit (PROBE), Department of Biomedicine, University of Bergen, Jonas Liesvei 91, Bergen 5009, Norway

${ }^{6}$ Department of Applied Mathematics, Computer Science, and Statistics, Ghent University, 9000 Ghent, Belgium

${ }^{7}$ Center for Proteomics and Metabolomics, Leiden University Medical Center, Leiden, The Netherlands 
Table of Content

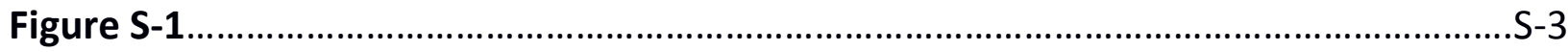




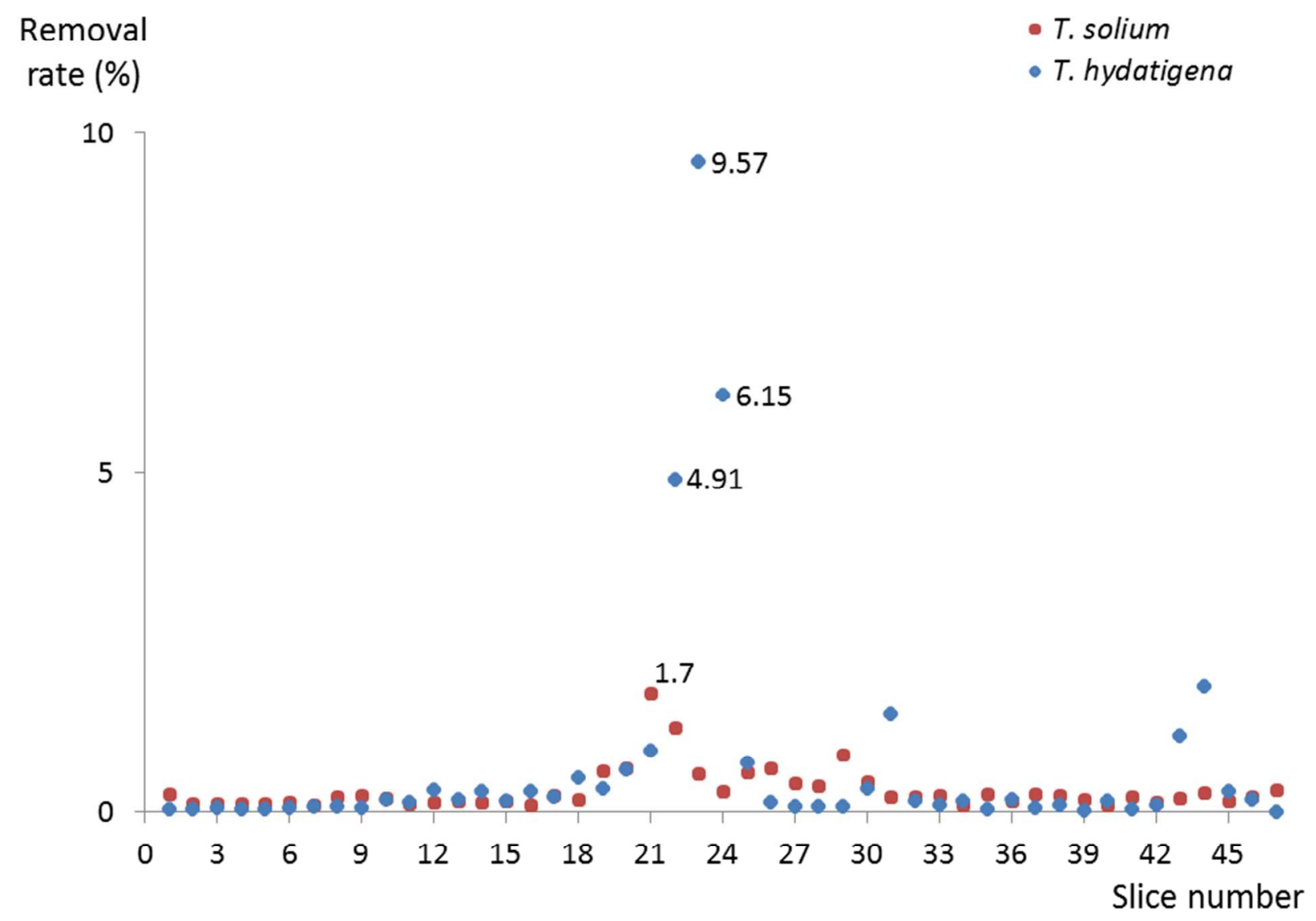

Figure S-1: Removal rates of MS/MS spectra per gel slice based on host and contaminant protein removal. The highest removal rates for Taenia solium and Taenia hydatigena were derived from slice number 21 (1.7\% of the MS/MS spectra in this slice), and slice number 23 (9.6\% of the MS/MS spectra in this slice), respectively. These slices correspond to the molecular weights of pig albumin (69.7 Da; 607 amino acids), and goat albumin (66.3 Da; 583 amino acids), respectively. 